\title{
Impact Analysis of the Hepatitis C Virus F Protein Deletion on Viral Replication and Pathogenic Infection
}

\author{
Hua Cao, Jianhua Gao, Xiaolong Liu \\ Jiangxi Medical College, Shangrao, Jiangxi, China, 334000
}

\author{
Keywords: Hepatitis C Virus; Core Protein; Viral Replication; F Protein
}

\begin{abstract}
Objective: investigating the influence of the hepatitis $\mathrm{C}$ virus $\mathrm{F}$ protein deletion on viral replication and pathogenic infection and the results were analyzed. Methods: the experimental method selected biochemical methods, the first step, the preparation of the hepatitis C virus RNA transcript; the second step, according to the result of the correlation detection culture experiments, including: PCR technology, the detection of infectious replication, immunofluorescence, transfection, etc. Finally, the relevant results to collate, record and analyze. Results: the introduction of the mutant gene expression and the number of the original number of genes expression was no significant difference in the expression levels of the two groups and the core protein also no significant difference $(\mathrm{P}<0.05)$; F protein expression in tumor tissue than other tissues, indicating HCC formation and F protein has some relevance; Core protein and the wild-type core protein contains a single mutation in the structure remained the same; F-deficient secondary structure of the core protein has more variations. Conclusion: HCV F translation and replication process of the missing protein of the virus had no effect on viral translation, reproduction and medical treatment to increase the resistance of the secondary structure of the core protein.
\end{abstract}

\section{Introduction}

Hepatitis $\mathrm{C}$ virus infection is the culprit leading to chronic liver disease and the resulting infection can be further developed into cirrhosis, fatty liver and liver cancer and other serious diseases [1]. There are about 170 million carriers of hepatitis $C$ virus worldwide, and about 100,000 people have developed cancer patients. It is generally believed that the route of transmission of hepatitis $\mathrm{C}$ virus includes blood and blood products, the current drug for hepatitis $\mathrm{C}$ virus treatment only interferon and the drug's response rate is very low, side effects are more expensive, does not meet the requirements, and Hepatitis $C$ virus vaccine is still in the research stage [3]. The hepatitis $\mathrm{C}$ virus genome consists of two parts, which are located in the middle of the open reading frame and at both ends of the non-coding region (conservative), the length can reach 9600bp. The noncoding region is indispensable for the viral RNA replication process and the translation process [4-5]. A single open reading frame translates as many as 3,000 amino acid residues, which are polyprotein precursors, which can be cleaved into six nonstructural proteins and 10 species under the dual action of viral proteases and host cells Structural proteins (E1, E2, C and P7). This year, some laboratories have found a viral protein called the reading frame shift protein, also known as core +1 protein. In addition, there are also found that the endogenous codon can also produce $\mathrm{F}$ protein, the starting site is mainly located in the $85 / 87$ codon was named core $+1 / \mathrm{S}$ [6]. From the discovery of F protein to now has more than ten years of history, but the human biology of its red to know little about how to understand the hepatitis $\mathrm{C}$ virus $\mathrm{F}$ protein is how the virus infection, disease and replication process, the study observed the process of viral translation and replication in the absence of $\mathrm{F}$ protein, and further analyzes how the secondary structure of the core protein changes after its deletion.

\section{Materials and Methods}

Reagents and Instruments. The name of the reagent and the instrument and the details of the 
manufacturer are shown in Table 1.

Table 1 Reagent and instrument name and manufacturer

\begin{tabular}{|c|c|}
\hline Reagent or instrument name & Production company or individual \\
\hline QuikChang LinghrningSite-Directed Mutagenesis & Stratagene Corporation (USA) products \\
\hline \multicolumn{2}{|l|}{ Kit } \\
\hline Isopropyl thiogalactoside & Beijing tripod biotechnology limited liability company \\
\hline 5-bromo-4-chloro-3-indole- $\beta$-D galactoside & Beijing tripod biotechnology limited liability company \\
\hline NZ amines & Beijing tripod biotechnology limited liability company \\
\hline Yeast extract & Beijing tripod biotechnology limited liability company \\
\hline 14ml BD Falcon Polypropylene Tube & Shanghai Ya Yi Science Experimental Equipment Co., Ltd \\
\hline HCV NS5A monoclonal antibody & Charles M .Riceprofessor \\
\hline T7 in vitro transcription kit & Promrga company \\
\hline M-MLV reverse transcriptase & Promrga company \\
\hline Fetal bovine serum & America invitorgen company \\
\hline DMEM & America invitorgen company \\
\hline Opti-MEM & America invitorgen company \\
\hline Alex488 goat anti-mouse fluorescent & America invitorgen company \\
\hline FITC-goat anti-human IgG & America invitorgen company \\
\hline Lipofectamine 2000 & America invitorgen company \\
\hline RNA extraction reagent & Shanghai Huashun Biological Engineering Company \\
\hline
\end{tabular}

Construction of Cell Lines and Plasmids. The introduction of the kit was carried out in the presence of PFL-J6JFH1 as template, and the number of introduced mutations was 5, and the purpose of introducing these mutations was to terminate the replication of all known F proteins, and this process did not Change the amino acid sequence of hepatitis $C$ virus.

Preparation of Viral RNA Transcripts. In the first step, the linearization treatment: the plasmid is linearized by XbaI; the second step, in vitro transcription: the preparation of RNA in vitro transcripts by T7 transcription kit; the third step, purity and integrity detection: reagent A mixture of phenol, isoamyl alcohol and chloroform at a volume ratio of 25: 1: 24 was selected and the purity and integrity of the mixture were tested by electrophoresis with $1.5 \%$ agarose gel.

RNA Transcript Transfection and Cell Culture. Transfection of RNA transcripts: $1000 \mathrm{~g}$ of trypsin digested cells were washed and centrifuged, separated, washed, resuspended, diluted, mixed and clicked $(270 \mathrm{~V}, 100 \mathrm{~m} \Omega)$ and then transferred to a constant temperature incubator $\left(5 \% \mathrm{CO} 2,37^{\circ}\right.$ C).

Cell culture: The culture medium, additives and conditions required for transfection are shown in Table 2. 
Table 2 Transfection of the desired culture medium, additives and conditions

\begin{tabular}{|c|c|c|c|c|c|c|c|c|c|}
\hline \multirow[b]{2}{*}{$\begin{array}{l}\text { Cell } \\
\text { name }\end{array}$} & \multicolumn{9}{|c|}{ Name of the culture medium, conditions, and additives } \\
\hline & $\begin{array}{l}\text { Culture } \\
\text { medium }\end{array}$ & temperature & $\mathrm{CO}_{2}$ & $\begin{array}{l}\text { Fetal } \\
\text { bovine } \\
\text { serum }\end{array}$ & $\begin{array}{l}\text { L-gluta } \\
\text { mine }\end{array}$ & NEAA & Streptomycin & penicillin & Incubator \\
\hline $\begin{array}{l}\text { Huh7. } \\
5.1\end{array}$ & DMEM & $37^{\circ} \mathrm{C}$ & $5 \%$ & $10 \%$ & $\begin{array}{l}2 \mathrm{mmol} \\
/ \mathrm{ml}\end{array}$ & $\begin{array}{l}0.1 \mathrm{mmol} \\
/ \mathrm{L}\end{array}$ & $100 \mathrm{~g} / \mathrm{ml}$ & $100 \mathrm{U} / \mathrm{ml}$ & $\begin{array}{l}\text { Saturation } \\
\text { humidity }\end{array}$ \\
\hline
\end{tabular}

Quantification of PCR. The specific primers were introduced into the non-coding region of HCV5 'and transfected. After three days, the total RNA of the transfected cells or the supernatant RNA was extracted to quantitatively detect the hepatitis $C$ virus RNA in the cells. The concentration of hepatitis C virus RNA was calculated.

Fluorescence Observation. The transfected cells were cultured and cultured on a cell culture plate (96-well cell culture plate) and then incubated in an incubator ( $\left.37^{\circ} \mathrm{C}, 5 \% \mathrm{CO} 2\right)$. After the culture was complete (about 48 hours) Discard the supernatant; add about 100 ul of methanol to each well and allow to stand for about $20 \mathrm{~min}$ in an environment at $-20^{\circ} \mathrm{C}$; wash with PBS for 5 minutes each time, then add Triton X-100 (1: 100), FITC-goat anti-human (1: 100) or Alex488 goat anti-mouse fluorescence (1: 100), followed by darkness feeding (1) Hour), and finally a fluorescence microscope to check and take pictures.

Virus Titer Detection. The cell supernatant was collected at different times and centrifuged at about $3000 \mathrm{~g}$ to remove the cell debris, and then stored at $-80^{\circ} \mathrm{C}$. Into the incubator to continue culture, and finally the virus titer detection.

Statistical Analysis. The data of the study were collated and collated. The comparison between the measurement data and the technical data was carried out by using the $t$ test and the $\chi^{2}$ test. The data were sorted out and the statistical analysis showed that EXEL and spss16.0 were different, respectively, and the difference was statistically significant [7].

\section{Results}

There was no significant difference between the expression of the mutated gene and the expression of the original gene. There was no significant difference in the expression level of the two groups $(\mathrm{P}<0.05)$.

Table 3 Comparison of the number of introduced mutant genes with the original gene expression

\begin{tabular}{|l|l|l|}
\hline gene & J6 Core gene & $\mathrm{J} 6 \mathrm{C} \Delta \mathrm{F}$ (F protein gene deletion $)$ \\
\hline Core gene $(5.0 \times 105)$ expression & $3.6 \times 10^{5}$ & $3.7 \times 10^{5}$ \\
\hline P Value & $<0.05$ \\
\hline
\end{tabular}

F protein deficiency on the pathogenicity of the virus: F protein expression in the tumor tissue is higher than other tissues, indicating that the formation of liver cancer and $F$ protein has certain relevance.

The secondary structure of RNA on the translation of the virus, the process of replication and pathogenicity: a single mutation containing the core protein and wild-type core protein in the structure of the basic consistent; $F$ deletion of the core protein secondary structure has more Many changes.

\section{Discussion and Conclusion}

The F protein is produced by transcoding the core coding sequence of the hepatitis $\mathrm{C}$ virus. It is generally believed that the coding of the core protein and the sequence encoding the $\mathrm{F}$ protein overlap each other. The initiation site of the internal translation of the gene is thought to be codon 26 [8-11]. The coding position of the Core $+1 / \mathrm{S}$ protein (the shortest $\mathrm{F}$ protein) is a gene of the 
core protein coding region (85-87), which is considered to be the starting point for the internal translation of the $\mathrm{F}$ protein [12]. So far, there have been a variety of mapping proteins, but their biological function is still very clear [13-15].

In order to gain a better understanding of the biological functions of the $\mathrm{F}$ protein and to determine how it affects the replication, translation and pathogenesis of the hepatitis $C$ virus, the study was conducted on wild-type J6JFH1 and self-constructed mutants (J6JFH1 / $\Delta$ F) Contrast and analysis. The results showed that the expression level of the protein of the mutant hepatitis $\mathrm{C}$ virus was much lower than that of the previous one. At the same time, the level of the RNA of the virus also decreased significantly (decreased by nearly 95\%), and the virus particles in the supernatant the number is also significantly reduced [16].

The RNA of the mutant RNA was transfected into Huh7.5.1 cells, and the expression of intracellular virus protein was detected. The titer of the virus RNA and virus in the supernatant were detected. The results showed that the wild type and mutant there is no significant difference between them [17]. Since the introduction of mutations may lead to changes in the secondary structure of the hepatitis $C$ virus core gene, the secondary structure of the core gene can influence the process of replication and translation of hepatitis $C$ virus RNA; further the secondary structure of the core gene Analysis, the results show that the process of translation and replication of the virus by the core gene secondary structure changes; the specific process to be further studied [18].

In summary, the virus replication, translation, pathogenicity and F protein deletion or not, but the core gene changes in the secondary structure will cause the virus translation and replication process changes, there is a correlation between the two.

\section{References}

[1] Feng L, Zhao Jinliang, Zhou Yulin, Ge Zhijun, Tang Shuiti, Wang Jiajia, Xu Ke, Wang Jie, Zhang Yun.Clinical level of IL-10 in plasma and its relationship with F protein [J]. Chinese Journal of Public Health, 2014, 30 (08): 1080-1081.

[2] Song Xiao-yan, Du Hong-mei, Yang Li-zhen, Meng Xiang-ying, Jia Li-ting, ZHANG Zhan.Study on prevalence and epidemiological characteristics of hepatitis $C$ virus infection in 68125 women of childbearing age [J]. Chinese Journal of Health Laboratory Technology, 2014, 24 (16): 2377-2379

[3] Xue Hongli, Feng Guohe, Dou Xiaoguang. Hepatitis C virus infection experimental model of new progress[J]. World Chinese Journal of Digestion, 2011, 19 (12): 1269-1274.

[4] Shi Wenfang, Feng Yue, Wei Daqiao, Xia Xueshan. Hepatitis C virus targeting drug and antiviral drug screening[J]. Chinese Journal of Biotechnology, 2011, 31 (11): 95-101.

[5] Faisal Nouroz, Sidra Shaheen .An overview on hepatitis C virus genotypes and its control Review Article Egyptian Journal of Medical Human Genetics, Volume 16, Issue 4, October 2015, Pages 291-298

[6] Shin Young Park, An-Na Kim, Ultraviolet-C efficacy against a norovirus surrogate and hepatitis A virus on a stainless steel surface International Journal of Food Microbiology, Volume 211, 15 October 2015, Pages 73-78

[7] Chen Jimei, Xu Yehong, Ding Xuefang. Chronic hepatitis C infection in a number of indicators of dynamic monitoring results analysis[J]. Chinese Journal of Disease Control, 2013, 17 (06): 548-550.

[8] Qi Yuying, Lin Zhenzhong, Ming Desong. IL-6, IL-10, IL-17 in patients with chronic hepatitis B blood levels[J]. Chinese Journal of Immunology, 2013, 29 (11) : 1177-1180.

[9] Xu Xiaodong, Lu Weidong, Yue Ming, Deng Xiaozhao, Zhu Danyan, Yu Xiaojie, Zhou Zhenxian, Ding Weiliang, Zhang Jinhai, Kong Jing, $\mathrm{Xu} \mathrm{Xu}$, Zhang Yun, Chu Chunli. Clinical 
significance of $\mathrm{F}$ gene variation in patients with hepatitis $\mathrm{C}$ virus infection. [J]. Chinese Journal of Disease Control, 2014, 18 (04): 322-325.

[10] Sericea Stallings-Smith, Kevin R. Krull, Long-term follow-up for incident cirrhosis among pediatric cancer survivors with hepatitis C virus infection Journal of Clinical Virology, Volume 71, October 2015, Pages 18-21

[11] Zhao Xiaonan, Li Hongyan, Bai Zhenzi, Zhao Hongyu, Sheng Chuanlun. 109 cases of hepatitis $\mathrm{C}$ virus infection in patients with metabolic syndrome clinical analysis[J]. Chinese Journal of Laboratory Diagnosis, 2011, 15 (11): 1866-1868

[12] Sun Ruina, Zhang Yan Ni, Wang Jun, Liu good orange, Kong Lingbao. [J]Chinese Journal of Pharmacoepidemiology, 2013, 48 (09): 1390-1396.

[13] Zhu Danyan, Lu Weidong, Deng Xiaozhao, Yue Ming, Xu Xiaodong, Shi Zhanying, Zhang Jiandong, Ding Weiliang, Zhang Jinhai, Xiao Wen, Pei Jiaping, Li Bingjun. Hepatitis C virus F protein up-regulated human peripheral blood mononuclear cells IL-13 expression [J]. Chinese Journal of Disease Control, 2015,19 (02): 115-118 + 126.

[14] Permission, Tang Fenyang, Wang Haopeng, Wang Jie, Yu Rongbin, Deng Xiaozhao, Zhang Yun. Analysis of F protein Antibody in Hemodialysis and Hepatitis C Virus Infection[J]. Chinese Journal of Public Health, 2012, 28 (03): 333-335.

[15] He Xiaolin, Huang Jianwei, Xu Ruian, Cui Xiuling. HBV replication mechanism and chronic hepatitis B drug target [J]. Chinese Journal of Pharmacology, 2015,31 (02): 152-156.

[16] Qi Yue, Jin Qinglong, Wen Xiaoyu, Niu Junqi. [J] Comparison of Replication Level of Hepatitis C Virus JFH1 in Different Three-dimensional Culture System [J]. Journal of Jilin University Medicine Edition, 2012,38 (01): 19-22 + 175.

[17] Wang Xin, Gao Xiangcui, Shi Wenwen, Zhang Guomin. Chronic hepatitis C treatment of individual anti-viral treatment [J] .Guangdong Medical, 2012,33 (10): 1516-1518.

[18] Li Xinxin, Lin Chunmin, Tian Shufang. Hospital health education in middle-aged and elderly patients with chronic hepatitis $\mathrm{C}$ application of Paroxin combined with ribavirin treatment compliance [J]. Chinese Journal of Gerontology, 2011, 31 (15) : 2866-2867. 